\title{
HISTORY AND EXAMINATION
}

\author{
A R Elkington, P T Khaw
}

\section{History}

\section{Visual symptoms}

- Monocular or binocular

- Type of disturbance

- Rate of onset

Presence and type of field loss

Associated symptoms-for example, flashing lights, floaters

- Effect on lifestyle

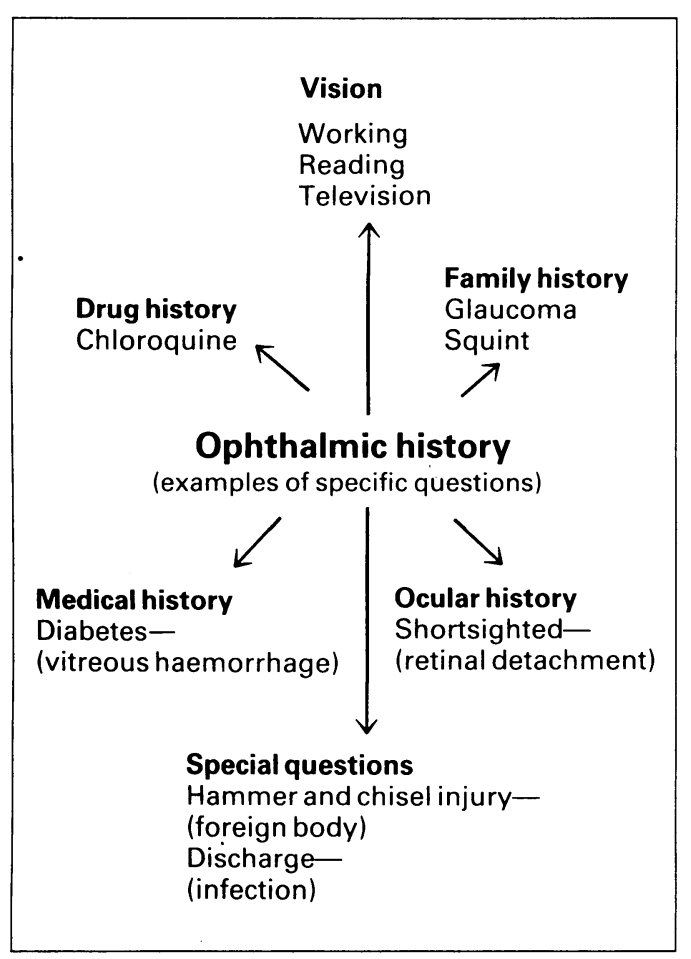

As in all clinical medicine, an accurate history and examination are essential if the correct diagnosis and treatment are to be achieved. Most ocular conditions can be diagnosed with a good history and simple examination techniques.

The history may give many clues to the diagnosis. Important points in the ophthalmic history obviously include visual symptoms.

The rate of onset of visual symptoms gives an indication of the cause. A rapid deterioration in vision tends to be vascular in origin, whereas a gradual onset suggests a cause such as cataract. The field of visual loss may be characteristic, such as the central field loss of macular degeneration. Symptoms such as flashing lights may indicate traction on the retina and impending retinal detachment.

It is particularly important to assess the effect of the visual disability on lifestyle, and difficulties with work, reading, watching television, and managing in the house should be identified. This is important in assessing the impact of the visual disability on the patient, especially when a condition such as cataract can now be operated on at an early stage with modern techniques.

The patient should also be asked exactly what worries him as visual symptoms often cause great anxiety. Appropriate reassurance can then be given.

\section{Questions about the particular symptom}

Some specific questions are important in certain circumstances. If there has been ocular trauma a history of any high velocity injury-particularly a hammer and chisel injury - should suggest an intraocular foreign body. Other questions-for example, about the type of discharge-may allow the diagnosis to be made in a patient with a red eye.

Ocular history is easily forgotten but is essential. The patient's red eye may be associated with contact lens wear. A history of severe shortsightedness (myopia) considerably increases the risk of retinal detachment. Patients often forget to mention eye drops and eye operations if they are just asked about "drugs and operations."

Medical history-Many systemic disorders affect the eye, and the medical history may give clues to the cause of the problem-for instance, a vitreous haemorrhage in a patient with diabetes.

Family history-The best example of the importance of the family history is in the case of chronic open angle glaucoma. This may be asymptomatic until severe visual damage has occurred. The risk of the disease is 1:10 in first degree relatives, and the disease may be arrested at an early stage. A family history of squint is also a risk factor in the development of squint.

Drug history-Many drugs affect the eyes, and they should always be considered as a cause of ocular problems-for example, chloroquine may affect the retina. 


\section{Examination of the visual system}

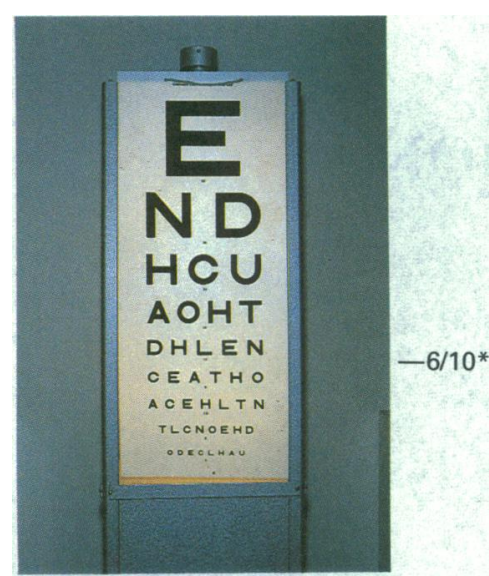

*Approximate level on Snellen chart required in at least one eye for driving a car.
Vision

An assessment of visual acuity is fundamental in any ocular disorder as it measures the function of the eye and gives some idea of the patient's disability. It may also have considerable medicolegal implications, as in the case of ocular damage at work or after assault.

Visual acuity is checked using a standard Snellen chart at six metres. If there is no room large enough a mirror can be used with a reversed Snellen chart at three metres. The numbers under the letters indicate the distance at which a person with no refractive error can read that line (hence the $6 / 60$ line should normally be read at 60 metres). If the top line cannot be discerned the test can be done closer to the chart. If the chart cannot be read at one metre patients may be asked to count fingers, and, if they cannot do that, to detect hand movements. Finally, it may be that they can perceive only light. From the patients' point of view the functional difference between these categories may be the difference between managing at home on their own (counting fingers) and total dependence on others (perception of light).

Vision should be tested with the aid of the patient's usual glasses or contact lenses. To achieve the optimal visual acuity the patient should be asked to look through a pin hole. This reduces the effect of any refractive error. It is particularly useful if the patient cannot use contact lenses because of a red eye or has not brought his glasses. If patients cannot read English they can be asked to match letters; this is also useful for young children.

Reading vision can be tested using a standard reading type book or, if this is not available, various sizes of newspaper print. There may be quite a difference in the near and distance vision. A good example is presbyopia, which usually develops in the late 40 s owing to the failure of accommodation with age. Distance vision may be $6 / 6$ without glasses but the patient may be able to read only larger newspaper print.

\section{Field of vision}

Testing the visual field may give clues to the site of any lesion and the diagnosis.

Location of the lesion - Unilateral field loss in the lower nasal field suggests an upper temporal retinal lesion. Central field loss usually indicates macular problems. A homonymous hemianopia indicates problems in the brain rather than the eye, though the patient may present with visual disturbance.

Diagnosis-If the patient has a bitemporal field defect this is most commonly due to a pituitary tumour. A field defect arching over central vision to the blind spot (arcuate scotoma) is almost pathognomonic of glaucoma.

To test the visual field - The patient should be seated directly opposite the examiner and should then be asked to cover the eye that is not being tested and to look at the examiner's face. If there is a gross defect the patient will not be able to see part of the examiner's face and may be able to indicate this precisely: "I can't see the centre of your face."

If no gross defect is present the fields can be tested more formally. Testing the visual field using finger movements peripherally will show severe defects, but a more sensitive test is the detection of red colour, because the ability to detect red tends to be affected earlier. A red pin is moved in from the periphery and the patient is asked when he can see something red. Finally, an extremely sensitive test is the comparison of the red in different quadrants. A good example is a patient who may have clinical signs of pituitary disease such as acromegaly; an early temporal defect can be detected if the patient is asked to compare the "quality" of the red in the upper temporal and nasal fields.
Move red pin in from periphery and ask patient to say when it is visible. 


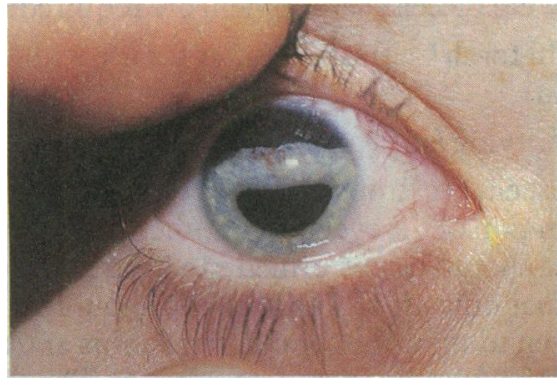

Torn peripheral iris (iridodialysis).

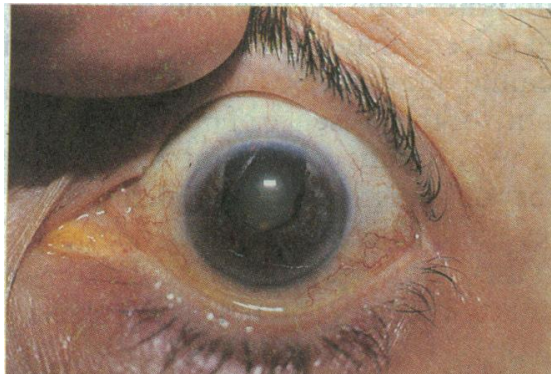

Distorted pupil after broad iridectomy.

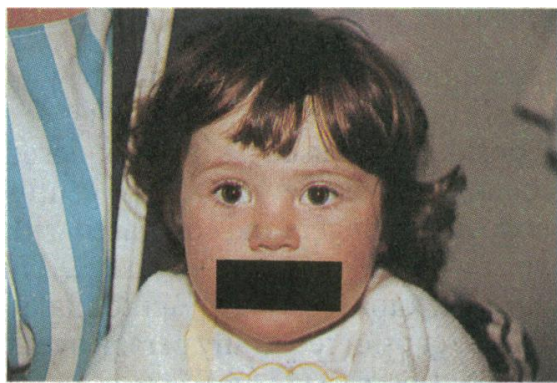

Normal position of corneal light reflexes.

\section{The pupils}

Careful inspection of the pupils can show signs that are helpful in diagnosis. A bright torch is essential. A pupil stuck down to the lens is due to inflammation within the eye, which is always serious. A peaked pupil after ocular injury suggests perforation with iris trapped in the wound.

The pupils' reactions to light are a simple way of checking the integrity of the visual pathways. By the time the pupils do not react to direct light, however, the damage is extremely severe. A much more sensitive test is the relative difference in pupillary reactions. The torch light is moved to and fro between the eyes not allowing time for the pupils to dilate. If one of the pupils does dilate when the light shines on it, there is a defect in the visual pathway on that side. Cataracts and macular degeneration do not usually cause an afferent pupillary defect unless the lesions are particularly advanced. Neurological disease must be suspected.

Other important and potentially life threatening conditions in which the pupils are affected include Horner's syndrome, where the pupil is small but reactive and there is associated ptosis. This condition may be caused by an apical lung carcinoma. The well known Argyll Robertson pupils caused by syphilis are rare. In a third nerve palsy the pupil is dilated and usually accompanied by ptosis; in this case the eye is divergent. Causes include a treatable intracranial aneurysm.

\section{Eye position and movements}

The appearance of the eyes will show the presence of any large degree of misalignment. This can, however, be misleading if the medial folds of the eyelid are wide. The position of the corneal reflections will help to confirm whether there is a true "squint." Squint and cover tests will be dealt with later in the series.

Patients should be asked if they have any double vision. If so, they should be asked to say whether diplopia occurs in any particular direction of gaze. It is important to exclude third (eye turned out) or sixth (failure of abduction) nerve palsies as these may be secondary to life threatening conditions. Complex abnormalities of eye movements should lead one to suspect myasthenia gravis or dysthyroid eye disease.

Test movements in all directions and also convergence

- Ask about double vision: if present, in which direction of gaze is it most pronounced?

Look for nystagmus

Eyelids: compare both sides and note position, lid lesions, and condition of margins

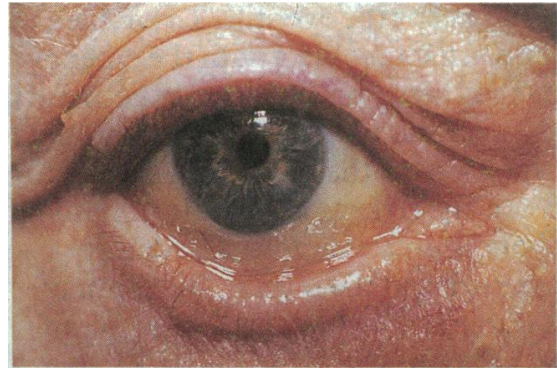

Ectropion.

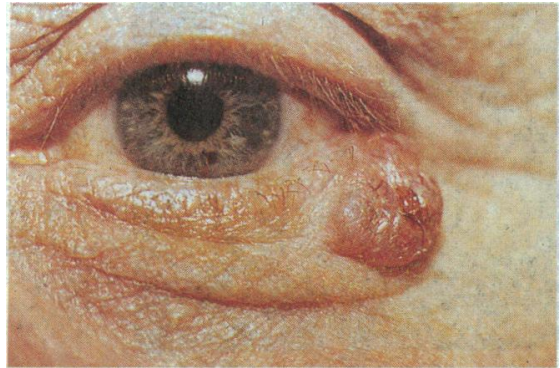

Basal cell carcinoma.

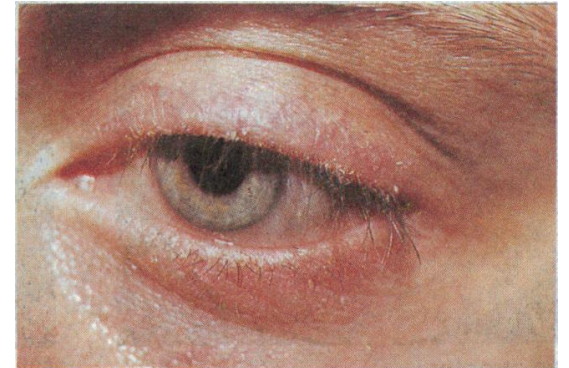

Blepharitis.

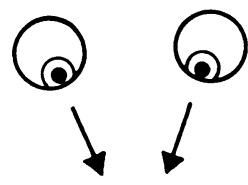

Convergence. 
Conjunctiva and sclera:

Look for local or generalised inflammation

Pull down lower lid and evert upper lid

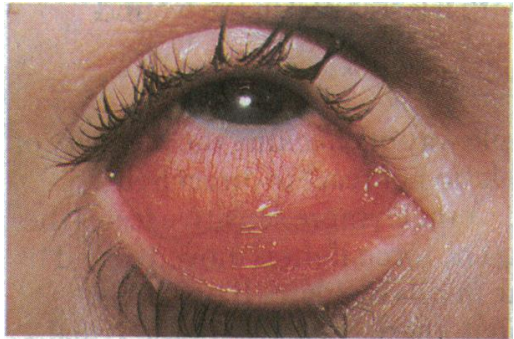

Conjunctivitis.

Cornea:

Look at clarity

Stain with fluorescein

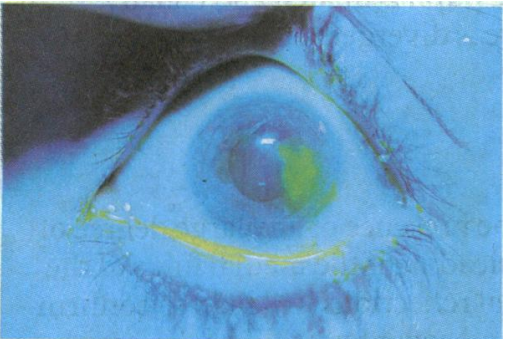

\section{Anterior chamber:}

- Check for blood and pus

Check chamber depth

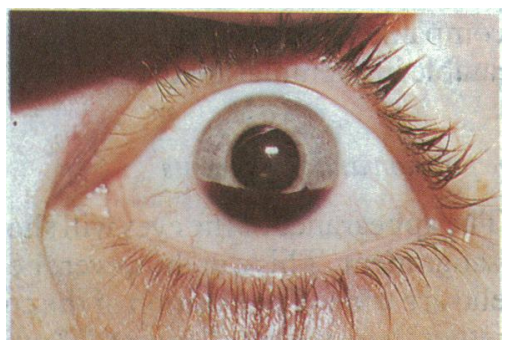

Corneal abrasion stained with fluorescein Blood in anterior chamber (hyphaema). and illuminated with blue light.

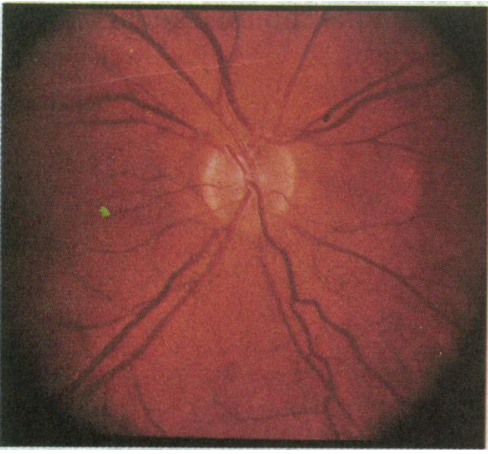

Normal disc.

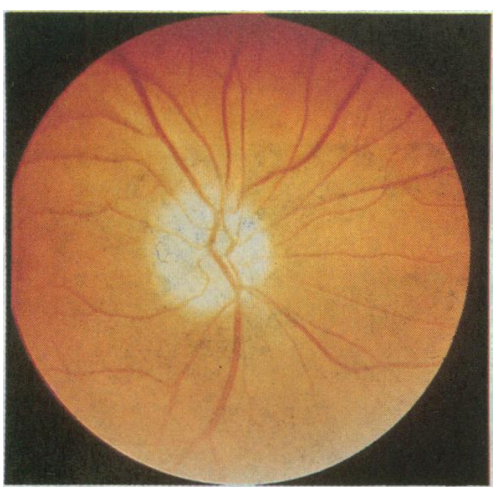

Optic atrophy.

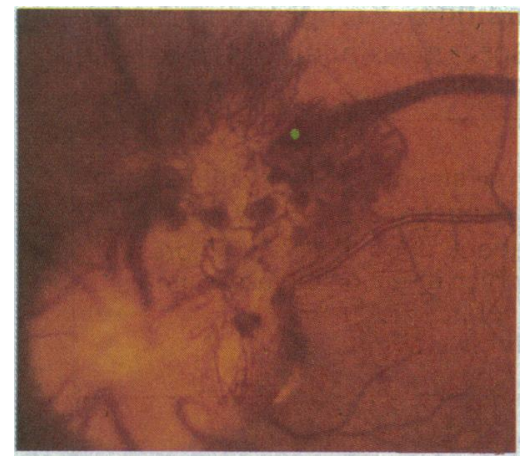

New vessels on optic disc in diabetes.

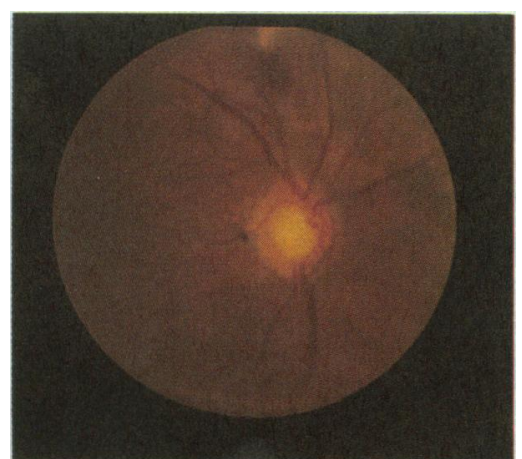

Glaucomatous cupping
The following pieces of equipment are required:

- A bright torch (with a blue filter for use with fluorescein)

- A magnifying aid

- Fluorescein eye drops.

Examination of the eyelids, conjunctiva, sclera, and cornea should be performed in a good light and with magnification. The lower lid should be pulled down to show the conjunctival lining and any secretions that may be in the lower fornix. The cornea should then be stained with fluorescein eye drops; if this is not done many lesions, including large corneal ulcers, may be missed. The anterior chamber should be examined looking specifically at the depth and for the presence of pus or blood.

If there are symptoms of "grittiness," a red eye, or any history of foreign body the upper eyelid should be everted. This should not be done, however, if there is any question of ocular perforation as the ocular contents may prolapse.

\section{Intraocular pressure}

The assessment of intraocular pressure by palpation is only useful when the intraocular pressure is considerably raised, as in acute closed angle glaucoma. The closed eye should be gently palpated between two fingers and compared with the other eye or with the examiner's own eye. The eye with acute glaucoma feels hard. Acute angle closure should be considered in any person over the age of 50 with a red eye.

\section{Ophthalmoscopy}

Good ophthalmoscopy is essential if many serious ocular and general diseases are not to be missed. To get a good view the pupil should be dilated. There is a risk of precipitating acute angle closure glaucoma, but this is small. Patients should be warned to seek help immediately if they have symptoms of pain or haloes around lights after having their pupils dilated. The best dilating drop is tropicamide $1 \%$, which is short acting and has little effect on accommodation. The effects may, however, still last several hours and the patient should be warned not to drive until any blurring of vision has subsided.

The ophthalmoscope should be set on the 0 lens. Patients should be asked to fix their gaze on an object in the distance as this reduces pupillary constriction and keeps the eye still. To enable a patient to fix on a distant object with the other eye the examiner should use his right eye to examine the patient's right eye, and vice versa. The light should be shone at the eye until the red reflex is elicited. This red reflex is the reflection from the fundus. If this is absent or diminished there is an opacity between the cornea and the retina. The commonest opacity is a cataract. 


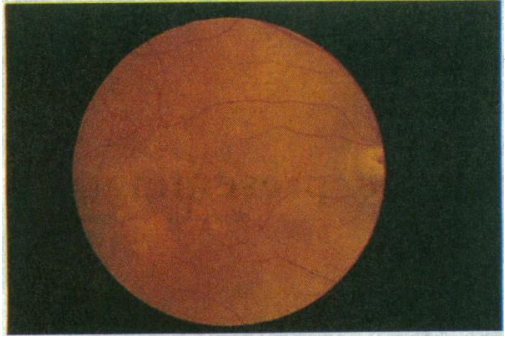

Senile macular degeneration.

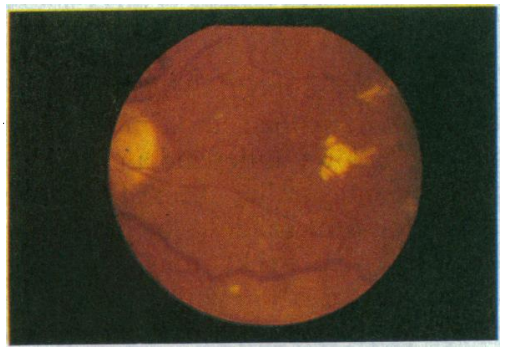

Diabetic maculopathy.
The optic disc should then be located and brought into focus with the lenses in the ophthalmoscope. If patients have high refractive errors they can be asked to leave their glasses on, though this can cause more reflections. The physical signs at the disc may be the only chance of detecting serious disease in the patient. A blurred disc edge may be the only sign of a cerebral tumour. Cupping of the optic disc may be the only sign of undetected chronic open angle glaucoma. New vessels at the disc may herald blinding proliferative retinopathy in a patient without symptoms. A pale disc may be the only stigma of past attacks of optic neuritis, or of a compressive cerebral tumour.

The retina should be scanned for abnormalities such as haemorrhages, exudates, or new vessels. The green filter on the ophthalmoscope helps to enhance blood vessels and microaneurysms. Finally, the macula should be examined for the pigmentary changes of senile macular degeneration and the exudates of diabetic macular degeneration.

Mr A R Elkington, FRCs, is senior lecturer in ophthalmology, Southampton Eye Hospital, and MrP T Khaw, FRCs, senior registrar at Moorfields Eye Hospital, London.

\section{ONE HUNDRED YEARS AGO}

Dr. Richter makes some observations in an article on this subject in the Berliner Klin. Wochenschrift, which ought to draw the attention of everyone concerned in the feeding of infants to this most important question. Most children in densely populated cities are brought up "by hand;" moreover, the number of those children who enjoy the natural provision of the mother's breast is steadily diminishing. There are several reasons for this; in the first place, there is the greater facility of artificial nourishment; secondly, the mother is often physically unable from her condition of health to nurse her child; and, thirdly, amongst the poorer classes the mother is often compelled by poverty to leave her home for the day in order to work. The best substitute for the mother's milk is that of some animal; this is proved by the immense number of artificial foods, including preserved milks, now competing for popular favour, and this number yearly increases, which shows that dissatisfaction still exists with regard to all such foods. Cow's milk lies under two chief objections: first, it is not easily digested by the stomach of an infant; and, secondly, it may harbour the most dangerous enemy of the human races, namely, tuberculosis. The chief cause of its comparative indigestibility is the character of the clot which it forms, owing to its containing more casein than human milk. This is to some extent remedied by the addition of a little barley water, amongst the intelligent, but there is a strong tendency to over-dilution of milk with water in recent years. With regard to tuberculosis, the cow is remarkably prone to this disease, for bovine tuberculosis (Perlsucht) is identical with tuberculosis in man; there can no longer be any doubt about this. The former is also much more common than the latter, though this is a truth little suspected by most people. Dr. Richter has been informed by veterinary surgeons that in some neighbourhoods as many as 50 per cent. of cattle die of tuberculosis, that the animal may show no signs of the disease during life, that no means of accurately diagnosing it are at present known, and that it is often clearly revealed only (though perhaps previously suspected) by a post-mortem examination. This is perhaps a pessimistic view, but it surely concerns us to know how the veterinary profession regard this subject.

It is not yet proved to general satisfaction that tuberculosis can be transferred by milk from the cow to human beings, though some authorities unhesitatingly believe that it can; at the same time, they tell us that any contained virus (of tuberculosis, or scarlatina, or other kind) is destroyed by the simple act of boiling the milk. It is a drawback to the use of milk that it should be regarded as a suspicious food requiring even this procedure, and, again, the young are more susceptible to infection than adults (as with the animals themselves), while their nourishment usually consists altogether of milk or mainly so for many years. It is easy to say that stringent sanitary regulations ensure the purity of milk, but it is always extremely difficult to ensure their being faithfully carried out; and, besides this, to do it at all effectively would entail vast expense upon the public at large. A still better mode, in Dr. Richter's opinion, is competition. The only animal that can compete with the cow is the goat, and on the whole goat's milk is far superior to that of the cow. To begin with, the goat is comparatively inexpensive, easy to keep in good condition, and far more easily kept clean than the cow. The goat will thrive almost anywhere, from mountain heights to the mews of a crowded city. Again, the goat, so far as is at present known, is rarely affected by tuberculosis; veterinary surgeons in neighbourhoods where goats abound tell us that this animal enjoys a high degree of immunity from that disease. In any case, as before remarked, it is far easier to control the sanitary surroundings of the goat than of the cow. Moreover, the putting to death of any suspicious animal will be effected with less hesitation in the case of the goat than with an animal costing fifteen or twenty times as much. And, lastly, the goat's milk itself, as is well known, is more easily digested by infants than cow's milk.

The analysis of Gorup Besanez stands thus: The quantity of water contained in 100 parts of human, cow's, and goat's milk respectively is $88.9,85.7,86.35$; of solids generally, $11.1,14.3,13.6$; of casein, 3.9, 4.8, 3.36; of albumen, a trace, $0.5,1.29$; of fat, 2.66, 4.3, 4.3 ; of milk sugar, $4.36,4,4$; of salts, $0.13,0.54,0.62$. Professor Fleischmann compares cow's with goat's milk as follows: There are of water 83.6 to 90 and 85 parts in cow's and goat's milk respectively; of fat, 2.8 to 4.5 and 4.8; of casein, 3.0 to 5.0 and 3.8 ; of milk sugar, 3.0 to 5.5 and 4 ; of albumen, 0.2 to 0.6 and 1.2; of ashy salts, 0.4 to 0.8 and 0.7 . These analyses agree in the main, and thus goat's milk, holding more albumen, is cheaper bulk for bulk than that of the cow. Taking everything into consideration, goat's milk could be provided at less cost than cow's milk. The chief advantage of the former lies in its ready assimilation by the digestive organs of the infant, owing to its relatively small proportion of casein, less than that of human, and much less than that of cow's milk.

It is not recommended that a sudden revolution should be attempted, and that the excellent milk now furnished to Londoners from the country should be exchanged altogether for that supplied by armies of goats. But in individual cases where cow's milk is not well tolerated by the child's system, it would be a great advantage if goat's milk could be conveniently substituted. It must be remembered, too, that a large proportion of the children of the poorer classes are fed with milk from cows kept in London. Such cows are largely fed with brewers' grains, and they produce a copious and watery milk, of unsatisfactory quality. They live under unnatural conditions, especially as regards total want of exercise, and it is not to be expected that they should enjoy perfect health.

When we consider the startling mortality amongst children under one year old in large cities, and the fact that the use of preserved milk is largely resorted to, and even advised indiscriminately by many medical men, it is strange that a source of milk so free from suspicion and so easily procurable as the goat should be so much overlooked. (British Medical foumal 1888;ii:25.) 